\title{
Effects of two treatments for aprosodia secondary to acquired brain injury
}

John C. Rosenbek, PhD; ${ }^{1-2 *}$ Amy D. Rodriguez, MA, CCC-SLP; ${ }^{1-2}$ Bethany Hieber, MA; ${ }^{1,3}$ Susan A. Leon, MA; ${ }^{1,4}$ Gregory P. Crucian, PhD; ${ }^{1,5}$ Timothy U. Ketterson, PhD; ${ }^{1,6}$ Maribel Ciampitti, MS; ${ }^{1,7}$ Floris Singletary, MS; ${ }^{1,8}$ Kenneth M. Heilman, MD; ${ }^{1,3,5-6}$ Leslie J. Gonzalez Rothi, PhD ${ }^{1,5,9}$

${ }^{1}$ Department of Veterans Affairs (VA) Rehabilitation Research and Development Brain Rehabilitation Research Center, Malcom Randall VA Medical Center (VAMC), Gainesville, FL; ${ }^{2}$ Department of Communicative Disorders, University of Florida, Gainesville, FL; ${ }^{3}$ Neurology Service, Malcom Randall VAMC, Gainesville, FL; Departments of ${ }^{4}$ Communicative Sciences and Disorders, ${ }^{5}$ Neurology, ${ }^{6}$ Clinical and Health Psychology, University of Florida, Gainesville, FL; ${ }^{7}$ University of Florida Health Sciences Center, Jacksonville, FL; ${ }^{8}$ University of Florida Brooks Center for Rehabilitation Studies, Gainesville, FL; ${ }^{9}$ Geriatric Research, Education, and Clinical Center, Malcom Randall VAMC, Gainesville, FL

\begin{abstract}
Expressive aprosodia is an impaired ability to change one's voice to express common emotions such as joy, anger, and sadness. Individuals with aprosodia speak in a flat, unemotional voice that often results in miscommunicated emotional messages. This study investigated two conceptually based treatments for expressive aprosodia: imitative treatment and cognitive-linguistic treatment. Five women and nine men with expressive aprosodia following right-hemisphere brain damage received the treatments in two phases 1 month apart in random order. Treatment was received 3 to 4 days a week for a total of 20 sessions each phase. As the outcome measure, sentences that elicited treated (happy, angry, sad, neutral) and untreated (fear) emotional tones of voice were administered during baseline, prior to treatment sessions, following treatment termination, and at 1- and 3-month followups. Effect sizes indicated that treatment effects were modest to substantial and that 12 participants responded to at least one treatment. Four responsive participants who were available for follow-up showed benefit at 1 and 3 months posttreatment. Most visual and statistical analyses were congruent.
\end{abstract}

Key words: aprosodia, cognitive-linguistic treatment, dysarthria, emotional prosody, expressive, imitative treatment, memory, receptive, rehabilitation, right-hemisphere damage, traumatic brain injury.

\section{INTRODUCTION}

Right hemisphere damage can affect a speaker's ability to produce and/or comprehend appropriate emotional prosody [1]. This condition is called aprosodia [2]. Expressive aprosodia is a speaker's inability to alter the intensity, frequency, duration, and quality of speech to nonverbally express an emotion. Receptive aprosodia is a listener's reduced ability to interpret another speaker's emotionally intoned speech [1]. Expressive and receptive aprosodia can co-occur or appear in isolation [1-2]. Despite the potentially disastrous effects on human relationships that aprosodia can cause, treatments are scarce, and until recently, single-patient reports were the only sources of data on treatment effects available to clinicians.

Anderson et al. investigated three treatments for expressive aprosodia secondary to right-hemisphere stroke in a 62-year-old man: a prosody repetition strategy, a cognitive-linguistic self-cueing strategy, and a facial

\footnotetext{
Abbreviations: ES = effect size, $\mathrm{FAB}=$ Florida Affect Battery, JOLO = Judgment of Line Orientation, MMSE = Mini-Mental Status Examination, Rey-O = Rey-Osterrieth Complex Figure Test, SLP = speech-language pathologist, $\mathrm{TBI}=$ traumatic brain injury.

*Address all correspondence to John C. Rosenbek, PhD; University of Florida, Department of Communicative Disorders, PO Box 100174, 101 South Newell Drive, Room 2150, Gainesville, FL 32611; 352-273-6170; fax: 352-2736545. Email: jrosenbe@phhp.ufl.edu

DOI: 10.1682/JRRD.2005.01.0029
} 
expression cross-cueing strategy [3]. The authors report that the prosody repetition strategy had the largest treatment effect. Stringer treated a 36-year-old woman with aprosodia from traumatic brain injury (TBI) [4]. He combined two treatments that he called pitch biofeedback and expression modeling. The pitch biofeedback component provided acoustic feedback via the Visipitch program. Expression modeling required the patient to imitate the clinician. The patient's prosody imitation and production were reported to improve.

More recently, we published two reports that detailed the visual and effect-size analyses of two treatments that were administered to four participants with primarily expressive aprosodia [5-6]. In both studies, a singlesubject ABAC design ${ }^{*}$ was used in which the two treatments, one imitative and one cognitive-linguistic, were administered in random order. Both treatments were conceptually driven as described in a previous publication [5]. The imitative treatment was based on the hypothesis that motor programming and planning of the vocal elements critical to expression of emotional prosody are impaired [7]. The hypothesis of an impaired modality-specific, nonverbal affect lexicon was the basis for the cognitivelinguistic treatment [8]. Effect sizes (ESs) for the two treatments across the two studies ranged from a modest 0.66 to a robust 11.51 . Based on visual analysis, three experienced clinician researchers concluded that both treatments positively affected all participants' expression of emotional prosody in sentence-length utterances.

Despite some similarities in treatment across the four studies described [3-6], confident conclusions cannot be made about the comparative effects of different treatments or the durability of improvement once treatment is concluded. Therefore, 10 subjects with expressive aprosodia and the 4 subjects who participated in the earlier studies [5-6] were treated with the design described in Rosenbek et al. [5]. Seven participants received both treatments and three participants (for various reasons to be described) received only one. This article summarizes our treatment experience with all 14 participants and has three purposes: (1) to summarize the results of ES and visual analyses for the two treatments, (2) to compare the effects of the two treatments, and (3) to measure the retention of treatment effects at 1 and 3 months posttreatment.

\footnotetext{
* Study design in which $\mathrm{A}=$ nontreatment phase, $\mathrm{B}$ = first treatment
} phase, and $\mathrm{C}=$ second treatment phase.

\section{METHODS}

\section{Participants}

The 14 participants (5 women and 9 men) were righthanded and, with one exception, were native speakers of American English. Participant 11 was born in Guyana (a former British colony) and his first language was also English. Ten participants had experienced a single, rightbrain cerebral infarction. Participant 14 was aprosodic following a cerebral hemorrhage, and participant 6 sustained a TBI during a motor vehicle accident. In addition to a right-hemisphere cerebral infarction, participant 10's imaging records also showed evidence of a left-hemisphere infarction, although this diagnosis was unknown at the time of informed consent. Ten participants had cortical lesions, five of whom also had extension into subcortical regions as measured by computed tomography scans. Three participants had lesions that were confined to subcortical structures and localization was unavailable on one. Time from stroke to enrollment in the study ranged from 4 months to 8 years. Of the 14 participants, 7 were being treated for depression. Table 1 summarizes the participants' demographic information.

\section{Recruitment and Screening}

Participants were recruited from four sites in northern Florida. Speech-language pathologists (SLPs) at all four sites identified potential participants in accordance with Health Insurance Portability and Accountability Act guidelines. The initial screening procedure for potential participants included a 20 -item task that assessed the individual's comprehension and production of emotional prosody, and a videotaped conversation between the individual and a caregiver about emotional experiences and personal photographs. In addition, three unpublished questionnaires (professional opinion questionnaire, therapist questionnaire, and caregiver questionnaire) were completed by the SLP and the caregiver for additional measures of each individual's emotional prosody. If an individual scored 14 or less on the 20-item screening task and at least one respondent to the three questionnaires judged the individual to be impaired in emotional prosody, four authors independently viewed the videotaped conversations. Study enrollment was then offered to any individual who was judged to be aprosodic by at least three of the four judges. Prior to the beginning of the study, the participants' informed consent was obtained in accordance with a protocol approved by the Health Sciences University of Florida 
Table 1.

Participant demographic information.

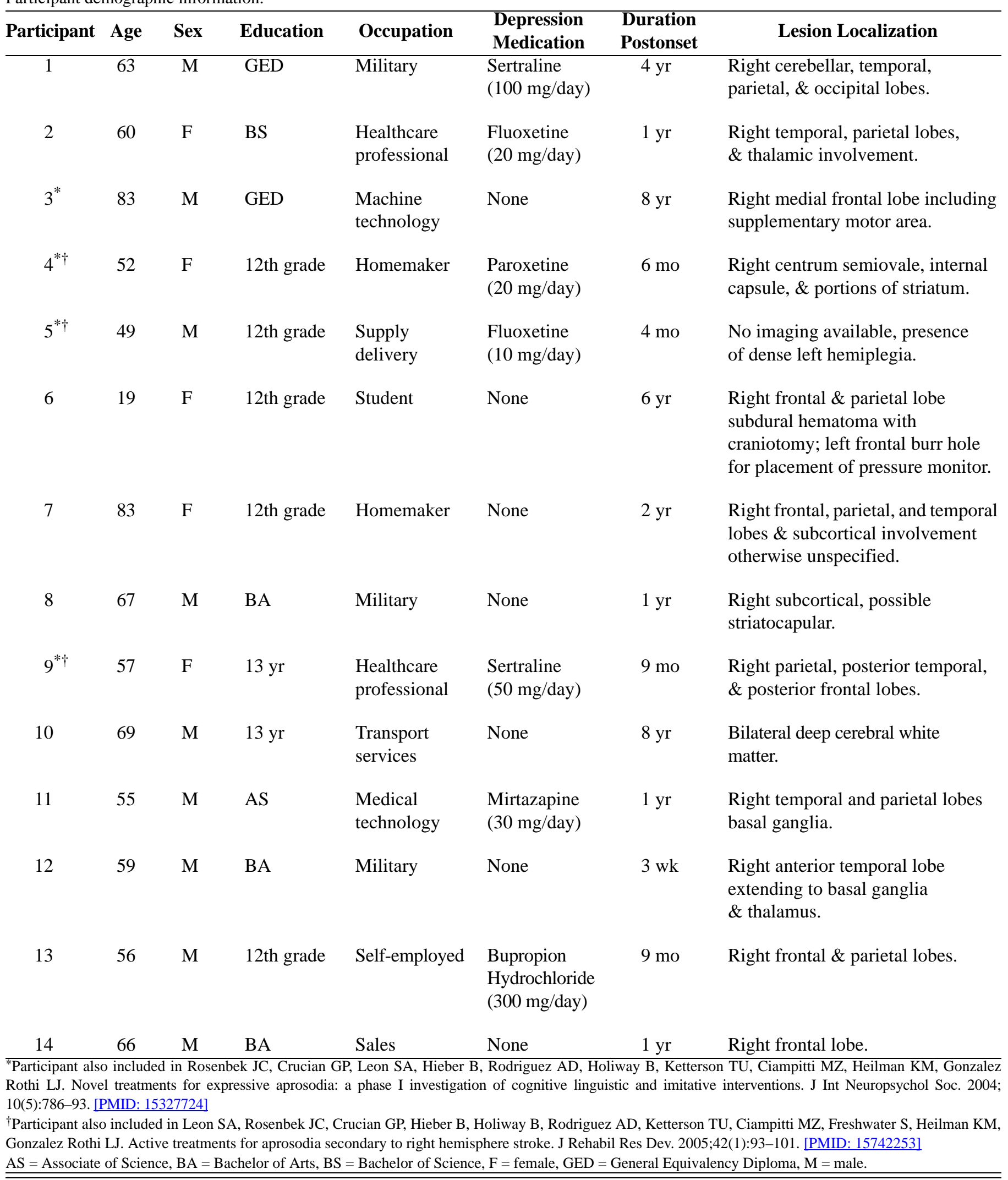


Center Institutional Review Board and the Subcommittee for Clinical Investigation at the Malcom Randall Department of Veterans Affairs Medical Center.

\section{Testing}

Once enrolled, participants who were able and willing to travel to the central study coordination site completed a battery of general cognitive tests as well as speech and prosody-specific assessments. Participants who were unable or unwilling to travel were visited in their residences and given an abbreviated battery that included, at a minimum, the speech and prosody-specific assessments. To screen for dementia, we administered the Mini-Mental Status Examination (MMSE) [9]. Visuospatial disorders were assessed using the Rey-Osterrieth Complex Figure Test (Rey-O) [10] and the Judgment of Line Orientation (JOLO) [11]. To assess for depression, we administered the Geriatric Depression Scale [12]. No participants were excluded because of performance on these measures and a variety of performance profiles emerged. A long-range goal of this research is identification of the variables that predict treatment response, and performance on these measures is anticipated to be among these predictive variables. Table 2 summarizes participant performance on these measures.

Of the 13 participants who completed the MMSE, 10 scored within normal limits. Three of nine participants scored within normal limits on the JOLO. The remaining participants (participants 1, 2, 4, 9, 10, and 13) were either impaired, borderline-impaired, or unable to complete the JOLO. Of the eight participants who completed the JOLO line-bisection task, four (participants 2, 4, 7, and 10) showed an attentional bias. Line-cancelation performance was generally within normal limits, although one participant (participant 2) exhibited more than mild difficulty and two (participants 5 and 7) had mild difficulty. Participant performance on the Rey-O complexfigure copying task was also generally within normal limits, although performance varied considerably and five participants (participants 5, 7, 9, 10, and 14) scored in the deficient range.

Severity of dysarthria and of receptive and expressive aprosodia were also determined. The distinction between dysarthria and aprosodia deserves special discussion. In dysarthria, a variety of prosodic abnormalities may occur but almost never in isolation. Instead, they are accompanied by variable combinations of sound imprecision, hypernasality, dysphonia, and reduced loudness. In aprosodia, prosody is uniquely impaired. Of course, the two conditions can co-occur, as they did in participants 1, 5, 10, and 12 . The previously described videotaped samples of each participant engaging in conversation with a caregiver were evaluated by four judges for the presence of dysarthria. If dysarthria was present, the severity was judged as mild, mild-moderate, moderate, moderate-severe, or severe.

Severity of receptive aprosodia was measured by performance on subtest 8A of the Florida Affect Battery (FAB) [13], in which participants identified the emotions

Table 2.

Summary of participants' neuropsychological testing.

\begin{tabular}{clccccc}
\hline Participant & \multicolumn{1}{c}{ JOLO } & Line Bisection & Line Cancelation & Rey-O & GDS & MMSE \\
\hline 1 & Borderline & -3.5 & $40 / 40$ & $15.75 / 36$ & 22 & $28 / 30$ \\
2 & Severely defective & -6.5 & $28 / 40$ & $23.5 / 36$ & 13 & $30 / 30$ \\
3 & DNT & DNT & DNT & DNT & 5 & $23 / 30$ \\
4 & Severely defective & -7 & $40 / 40$ & $29 / 36$ & DNT & $26 / 30$ \\
5 & Low average & -1 & $39 / 40$ & $12.5 / 36$ & 10 & $28 / 30$ \\
6 & Average & +1.5 & $40 / 40$ & $33 / 36$ & 12 & $27 / 30$ \\
7 & DNT & -9 & $39 / 40$ & $1.5 / 36$ & 9 & $27 / 30$ \\
8 & DNT & DNT & DNT & DNT & DNT & $25 / 30$ \\
9 & DNT & DNT & $40 / 40$ & $13.5 / 36$ & 10 & $27 / 30$ \\
10 & Severely defective & +13 & $40 / 40$ & $12.5 / 36$ & 12 & $22 / 30$ \\
11 & Average & DNT & $40 / 40$ & $34 / 36$ & 1 & $30 / 30$ \\
12 & DNT & DNT & DNT & DNT & 11 & $25 / 30$ \\
13 & Severely defective & +3 & $40 / 40$ & $20.5 / 36$ & 12 & $23 / 30$ \\
14 & DNT & DNT & $40 / 40$ & $6 / 36$ & 8 & DNT
\end{tabular}

DNT = did not test, GDS = Geriatric Depression Scale, JOLO = Judgment of Line Orientation, MMSE = Mini-Mental Status Examination, Rey-O = Rey-Osterrieth Complex Figure Test. 
expressed in 20 semantically neutral sentences spoken in one of five affective tones of voice (happy, sad, angry, fearful, and neutral). Severity of receptive aprosodia was classified as follows:

- Normal $=$ FAB score $<1$ standard deviation (SD) below the mean.

- Mild = FAB score 1.0 to 1.5 SD below the mean .

- Moderate $=$ FAB score 1.5 to 2.0 SD below the mean .

- Severe $=$ FAB score $>2$ SD below the mean.

Three of the four judges had to agree on the severity score.

We used the previously described videotaped conversation plus a tape-recorded performance on an unpublished expressive emotional communication battery (under development at the Cognitive Neuroscience Laboratory at the University of Florida) to establish the severity of expressive aprosodia. The unpublished expressive emotional communication battery assesses a person's ability to imitate syntactic and emotional prosody and produce syntactic and emotional prosody to command. After reviewing the participant's videotape and taperecording, the same four judges independently assigned a severity score (mild, mild-moderate, moderate, moderate-severe, or severe). Three of the four judges had to agree on the severity score. Table 3 summarizes the severity of dysarthria, receptive aprosodia, and expressive aprosodia for all participants.

\section{Experimental Design}

We used a single-subject ABAC design with replication across 14 participants. During the initial nontreatment

Table 3.

Severity of participants' dysarthria and aprosodia.

\begin{tabular}{clll}
\hline \multirow{2}{*}{ Participant } & \multirow{2}{*}{ Dysarthria } & \multicolumn{2}{c}{ Aprosodia } \\
\cline { 3 - 4 } & & Receptive & Expressive \\
\hline 1 & Moderate & Mild & Severe \\
2 & None & Severe & Moderate \\
3 & None & Severe & Moderate-severe \\
4 & None & Moderate & Moderate \\
5 & Mild & Normal & Moderate \\
6 & None & Normal & Severe \\
7 & None & Moderate & Moderate \\
8 & None & Severe & Severe \\
9 & None & Normal & Mild \\
10 & Mild-moderate & Severe & Moderate \\
11 & None & Normal & Mild \\
12 & Moderate & Severe & Moderate-severe \\
13 & None & Normal & Mild \\
14 & None & Mild & Severe \\
\hline \hline
\end{tabular}

phase (A), stable baselines for verbal production of five emotional tones of voice in sentence-length utterances were established and verified by the C-statistic [14]. The treatment phases (B and C) were approximately 1-month long and contained 20 treatment sessions; each session averaged 1 hour. The two treatments (imitative and cognitive-linguistic) were applied in random order. The second A, or nontreatment phase, was also 1-month long. Each treatment phase was immediately followed by two sessions of posttesting. Of the 14 participants, 5 also received follow-up testing at 1 month posttreatment and 7 received follow-up testing at 3 months posttreatment.

Seven participants were randomly assigned to receive imitative treatment during the first treatment period, followed 1 month later by cognitive-linguistic treatment. The other seven participants were randomly assigned to the opposite treatment order. Three participants dropped out of the study after receipt of only the first treatment: participant 6 received only imitative treatment and participants 12 and 13 received only cognitivelinguistic treatment.

\section{Treatment Procedures}

This study investigated two conceptually based treatments for expressive aprosodia: imitative and cognitivelinguistic. Both treatments followed a six-step cueing continuum in which the clinician provided maximum cueing in the first step and systematically decreased cueing as the participant progressed to the final step. In the imitative treatment, the clinician provided a model sentence with the target emotional prosody and the participant repeated the sentence and attempted to use the same emotional tone of voice. The participant moved from imitation of the model to independent production. In the cognitive-linguistic treatment, the participant was provided with three cards: one with the name of the target emotion, one with the vocal characteristics of the target emotional tone of voice, and one with a picture showing the corresponding facial expression. The participant was then given a sentence and asked to say it using the cues provided on the cards. The cards were systematically removed as the participant successfully produced each sentence. The steps for both experimental treatments and the stimuli used in each step are outlined in Appendix 1 and Appendix 2 (available online only at http:// www.rehab.research.va.gov/).

For both treatment methods, nine randomly selected sentences (three each of happy, sad, and angry) from the 
treatment set (further described in "Treatment Stimuli") were chosen by a predetermined rotational order and treated each session. While the study was also designed to include a neutral emotion in the treatment, all participants produced the neutral sentences with 100 percent accuracy in the baseline phase, so these sentences were not trained. Treatment began at step 1 for each treated sentence and the participant advanced after three consecutive correct responses at each step. If the participant failed to produce three correct consecutive responses after five attempts, the clinician returned to the previous step to elicit three consecutive correct responses. The clinician discontinued the sentence if it was necessary to return to a previous step more than twice.

\section{Treatment Stimuli}

Together with other colleagues, we developed the treatment and outcome-measure stimuli. Lists of sentences that invoked an affective response were compiled; the sentences that elicited the strongest affective response for each emotion among us and our colleagues were selected as stimuli. All sentences were semantically congruent with their accompanying emotional tone of voice. The sentences were divided into three sets: imitative treatment (20 sentences), cognitive-linguistic treatment (20 sentences), and one that was never treated but used to sample generalization (30 sentences). Additionally, a set of 10 sentences was used for eliciting linguistic prosody. A representative sample of the stimuli sentences used in this study can be found in Appendix 3 (available online only at http://www.rehab.research.va.gov/).

\section{Outcome Measure}

Treatment effect was measured by the administration, rating, and analysis of an outcome measure. The outcome measure consisted of sentences that elicited target emotional tones of voice for the four treated emotions (happy, sad, angry, and neutral) and one randomly selected nontreated control emotion (fear). These sentences were also used for experimental control (linguistic prosody and fear), generalization (eliciting trained tones of voice but never treated), and active treatment.

Two versions of this outcome measure were used: a 50-item version in the baseline and follow-up phases and a 45 -item version prior to each treatment session. The 50 item outcome measure consisted of 10 linguistic prosody sentences, 5 fearful sentences, 10 sentences from the set used during imitative treatment, 10 sentences from the set used during cognitive-linguistic treatment, and 15 sentences from the set that was never treated but used to sample generalization. The 45-item outcome measure was the same except that only five sentences from the current treatment were presented for reduced testing time. The sentences were rotationally ordered and balanced across emotions so that all sets were probed equally over the course of treatment.

\section{Outcome Measure Administration}

The 50-item outcome measure was administered a total of 24 times: 8 times during each pretreatment baseline phase, 2 times immediately after each treatment phase, 2 times at the 1-month follow-up, and 2 times at the 3-month follow-up. The 45-item version was administered prior to the start of each therapy session during both treatment phases. The clinician administered the outcome measure by presenting a sentence written on a card and instructing the participant to say the sentence aloud using a particular tone of voice (e.g., the patient was shown the sentence "I just won the lottery" and asked, "Please say this sentence using a happy voice").

\section{Outcome Measure Perceptual Analysis}

The participants' responses to the outcome measure were audiotaped. The first four participants were taped with a Marantz audio recorder (Itasca, Illinois); all subsequent participants were taped with a Tascam digital audio recorder (model DA-P1, Montebello, California). Each sentence was scored as "plus" if emotional prosody was correct and "minus" if incorrect. The clinician scored online during each session. For establishing reliability, a second trained judge who was blind to the time of testing later judged the adequacy of all responses from the audiotape. Both the clinician's and trained judge's assessments were based solely on verbal expression. This was accomplished by having the clinician look away while the participant produced each sentence, a condition that was explained to the participant in the first treatment session.

The blinded judge was an SLP with 2 years of experience in evaluating the prosody of emotionally intoned sentences. Training for this judge included familiarization with the descriptions of features for each emotion with respect to changes in pitch, loudness, and rate. The judge also took part in research group sessions in which tapes of aprosodic speakers were discussed and individual features were rated. 
Both intra- and interjudge reliability were calculated for the blinded judge with 20 percent of each participant's productions. Intrajudge reliability was acceptable (Kendall $\tau=0.75, p<0.001$ ). Interjudge reliability based on judgments by the blinded judge and another experienced clinician was also acceptable (Kendall $\tau=0.79$, $p<0.001)$. The blinded judge's scores, reported as percentage of prosodically correct responses, were the data used in all statistical analyses. The scores were graphed as shown in Appendix 4 and Appendix 5 for visual analysis (available online only at http://www.rehab.research.va.gov/).

\section{RESULTS}

Our first purpose was to determine if the two treatments were active. Visual and statistical analyses were used for this determination. Three SLPs with at least 3 years experience judging data via visual inspection completed the visual inspection of the outcome measure data. The judges independently assessed the stability of both baseline phases for each participant and then considered the relative slope and height of the data displays during the two treatment phases. The judges were given similar directions for judging displays of the untreated emotion. Appendix 5 displays the graphs that the judges used for the visual analysis (available online only at http:// www.rehab.research.va.gov/).

For the statistical analyses, ESs [15] were calculated for each participant and each therapy. The ES for the first treatment was calculated by subtraction of the mean of the correct responses on the eight baseline outcome measures from the mean of the correct responses on the twenty therapy outcome measures, divided by the standard deviation of the baseline outcome-measure data. The ESs for each participant for the second treatment were calculated identically with data from the second therapy. The formula for calculation of ES is

$$
\frac{M^{\text {therapy }}-M^{\text {baselines }}}{\text { SD }^{\text {baselines }}},
$$

where $M=$ mean and SD = standard deviation.

Three judges agreed that the visual displays of outcome measure data (Appendix 4, available online only at http://www.rehab.research.va.gov/) showed evidence of treatment effects from both treatments for six of the 11 participants who received both treatments (participants 2,
5, 7, 8, 9, 11). Participant 4 would also have been included but one judge reported no treatment effect for the imitative treatment. Of the 11 participants who received both treatments, 3 showed no treatment effect for one of the two treatments (participants 1 and 4 for cognitive-linguistic, participant 3 for imitative). All the judges reported that participant 10 showed no effect for either treatment. Participant 14's performance produced the most complicated outcome. Three of the judges reported no treatment effect for the imitative treatment and two of the three also reported no treatment effect for the cognitive-linguistic treatment. The judges determined that all three participants (participants 6, 12, and 13) who received only one treatment showed a treatment effect. No evidence of generalization to the untreated emotion (fear) was noted for any participant and no statistical analysis was completed; the lack of generalization was obvious in that no acquisition of the ability to indicate fear was present for either treatment for any participant.

The ESs ranged from a negative effect of -0.22 to a substantial positive effect of 11.51 . If one accepts Cohen's position that an ES of 0.6 is modest and 0.8 is robust [16], the statistical analysis results were mostly consistent with the visual analyses. Of the 25 treatments judged visually and statistically (3 of the 14 participants received only one treatment), 24 (96\%) of the analyses were consistent, which means that ESs $>0.6$ were judged significant by visual analysis. The exception occurred for Participant 3 for whom the judges did not report a treatment effect via visual analysis despite an ES of 1.22. Two additional, albeit slight, deviations in the data warrant mention. One of the three visual-analysis judges thought that participant 14's response to cognitive-linguistic treatment was positive; however, the ES was -0.22 . The other deviation occurred for participant 4 on the imitative treatment. The ES was 2.54 but one of the three judges did not perceive a treatment effect upon visual analysis. The results of the statistical and visual analyses are shown in Table 4.

Our second purpose was to compare effects of the two treatments using the visual and statistical analysis data. By visual inspection, we found similar patterns of treatment response regardless of treatment order. Of the seven participants who began with the imitative treatment (one received only this treatment), six had a linearly increasing percent correct on the treated emotions and one had no change. This pattern was essentially the same for the participants who received the cognitive-linguistic 
JRRD, Volume 43, Number 3, 2006

Table 4.

Results of statistical (effect size) and visual analyses of participants' responses to cognitive-linguistic and imitative aprosodia treatments presented in two phases 1 month apart and in random order.

\begin{tabular}{|c|c|c|c|c|c|c|c|c|c|c|}
\hline \multirow{3}{*}{ Participant } & \multicolumn{5}{|c|}{ Treatment Phase 1} & \multicolumn{5}{|c|}{ Treatment Phase 2} \\
\hline & \multirow{2}{*}{ Treatment } & \multirow{2}{*}{ Effect Size } & \multicolumn{3}{|c|}{ Visual Analysis* } & \multirow{2}{*}{ Treatment } & \multirow{2}{*}{ Effect Size } & \multicolumn{3}{|c|}{ Visual Analysis* } \\
\hline & & & $\mathbf{R 1}$ & $\mathbf{R 2}$ & $\mathbf{R 3}$ & & & $\mathbf{R 1}$ & $\mathbf{R 2}$ & R3 \\
\hline 2 & Cog-Ling & 5.01 & + & + & + & Imitative & 0.98 & + & + & + \\
\hline 3 & Cog-Ling & 1.22 & - & - & - & Imitative & 1.18 & + & + & + \\
\hline 4 & Cog-Ling & 0.66 & + & + & + & Imitative & 2.54 & + & + & - \\
\hline 6 & Imitative & 2.16 & + & + & + & No Tx2 & NA & NA & NA & NA \\
\hline 7 & Imitative & 3.02 & + & + & + & Cog-Ling & 0.62 & + & + & + \\
\hline 8 & Imitative & 2.64 & + & + & + & Cog-Ling & 0.84 & + & + & + \\
\hline 9 & Imitative & 3.68 & + & + & + & Cog-Ling & 2.76 & + & + & + \\
\hline 10 & Imitative & -0.06 & - & - & - & Cog-Ling & -0.01 & - & - & - \\
\hline 14 & Cog-Ling & -0.22 & - & - & - & Imitative & 0.53 & - & - & - \\
\hline
\end{tabular}

*+ = treatment effect, $-=$ no treatment effect.

Cog-Ling = cognitive-linguistic, NA = not applicable, $\mathrm{R}=$ rater, Tx2 = Treatment 2.

treatment first. In addition, the majority of cases showed that the treatment effect for the first treatment was larger than for the second regardless of which treatment came first. We also conducted a two-way analysis of variance with improvement from baseline to treatment as the dependent variable and treatment group and order as the two independent variables. No significant difference for treatment group or order emerged. Table 5 summarizes the gains by treatment group and order.

Our third purpose was to determine the retention or generalization of treatment effects across time. In other words, did participants maintain treatment effects 1 and 3 months after treatment termination? The necessary follow-

Table 5.

Treatment gains by treatment group and order.

\begin{tabular}{lcccc}
\hline \multicolumn{1}{c}{ Variable } & $\boldsymbol{n}$ & Gain \pm SD & Min & Max \\
\hline Group & & & & \\
$\quad$ Cognitive-Linguistic & 13 & $12.15 \pm 11.56$ & -0.05 & 41.30 \\
$\quad$ Imitative & 12 & $9.78 \pm 7.18$ & -0.23 & 25.29 \\
Order & & & & \\
1 & 14 & $11.26 \pm 11.63$ & -0.23 & 41.30 \\
2 & 11 & $10.70 \pm 6.69$ & -0.05 & 22.72 \\
\hline Max = maximum, Min = minimum, SD = standard deviation. & \\
\hline
\end{tabular}

up data were available for 6 of the 14 participants. To determine retention, we used $t$-tests that compared the percent correct responses from posttesting and the six immediately preceding sessions with the percent correct responses from the 1- and 3-month follow-ups (participants 2 and 9 only had 3-month data). Participants 2, 5, 7, and 9 had retained their gains, while participants 3 and 4 had not.

\section{DISCUSSION}

This sample of 14 aprosodic participants appears to be the largest number ever treated in an experimental protocol. Clearly, systematic replication and group studies with random assignment are necessary before confident treatment recommendations can be made. Nonetheless, single-case design methodology provides sufficient experimental rigor to support a discussion of these findings and reasonable directions for future programmatic research.

Perhaps of greatest clinical interest is that 12 of the 14 participants showed a statistically significant response to at least one of the treatments (ES >0.6). Thus, these data can become a critical component of evidence-based practice in expressive aprosodia. Of course, more data are necessary for confident decision making. Differences 
in the effects of the two treatments could not be discerned. However, a trend toward larger effects of the first treatment in participants who responded to both treatments was found, regardless of which treatment came first. These findings leave clinicians free to choose the treatment method with which they are most comfortable. Generalization to the untreated emotion (fear) did not occur. Thus, clinicians using either treatment method, depending on their goals, may want to treat a full array of emotional prosodic profiles. Additionally, they may want to experiment with more intensive treatment. Gains were retained for 3 months in the four participants whose data allowed analysis. If this evidence of retention can be confirmed in subsequent studies, clinicians can reasonably expect gains to persist for at least 3 months. In light of clinical pessimism about the successful treatment of neurobehavioral disorders after right-hemisphere strokes, these results are cause for optimism.

An analysis of individuals in this sample of treated participants contributes additional clinical information and supports the design of subsequent rehabilitation research. Consider, first, the nonresponders. All participants responded to both treatments except participants 10 and 14 who did not respond to either treatment and participant 1 who did not respond to the imitative treatment (which came first) but subsequently responded to cognitive-linguistic treatment.

Being able to predict whether an individual patient will respond to treatment is important to treatment planning. A number of factors, singly and combined, may contribute to a lack of response by aprosodic speakers. These factors include distribution and extent of brain damage, etiology, insight into deficit, depression, performance on the neuropsychological battery, severity of receptive and expressive aprosodia, presence of dysarthria, duration of aprosodia, and even past musical background. One of the three nonresponders (participant 10) had bilateral infarcts; therefore, one can hypothesize that he had reduced opportunity for improvement because he lacked a healthy hemisphere that was capable of assuming neural control of emotional prosody. All other participants, including the other two nonresponders, had unilateral lesions. Thus, bilateral brain damage may signal a poor prognosis but presence of unilateral damage is not necessarily a good sign either. One nonresponder (participant 14) experienced a hemorrhage that involved the right frontal lobe. No other participant experienced a hemorrhage but many of the responders had more exten- sive involvement. Participant 2, for example, had cortical and thalamic lesions. Participant 14's severely limited insight into his condition despite normal receptive prosody may have been a more important predictor of his nonresponse. At times he denied having had a stroke. Lack of insight was not so obvious in the other two nonresponders, however. Given the well-known anosognosia of persons with right-hemisphere damage, insight may have been limited in varying degrees for both nonresponders and responders. Unfortunately, this study did not include formal assessment of insight. Severity of receptive aprosodia, which was carefully measured as a surrogate for insight, is not a strong predictor of response. Participants 1 and 14 (both nonresponders) had severe receptive aprosodia but so did four of the responders. Insight measurement will be a part of diagnostic testing in our subsequent treatment studies.

The common clinical wisdom that depression predicts response to treatment in a variety of conditions was not confirmed in this study. Participants 10 and 14, who did not respond to either treatment, were not depressed. Participant 1 , who failed to respond to one of the two treatments, reported "struggling" with depression and frequently complained about the study. However, other participants, including participant 4 , who were also medicated for depression and complained about the treatments, did respond to treatment. The nonresponders were all judged to exhibit moderate-to-severe expressive aprosodia; however, severity alone did not determine response, since participants 6 and 8 also demonstrated severe expressive aprosodia yet responded to treatment. Dysarthria alone is not a predictor; it was present in two nonresponders and two responders. Performance on neuropsychological measures also did not reliably predict response. Participant 10 scored outside normal limits on three of the five neuropsychological measures, while participant 14 did so on only one. In contrast, participant 2 was outside normal limits on three of the five measures but showed an ES of $>5.0$ as a result of cognitive-linguistic treatment. Finally, even the influence of duration of aprosodia is muddled by this study's data. Participant 5, with an ES of 11.51, had been aprosodic for 4 months. In contrast, participant 12 had been aprosodic for only 3 weeks but responded with an ES of only 1.47 before withdrawing from treatment. The search for response predictors is complicated by the range of treatment ESs. At the low end is participant 7's second treatment (0.62, cognitive-linguistic) and participant 4's first treatment (0.66, cognitive-linguistic). Out of all 
participants across treatments, participant 5 showed the greatest ES (11.51) for the cognitive-linguistic treatment. He also responded robustly to the imitative treatment and reached ceiling criterion for discontinuation of treatment after 10 sessions (85\% total correct on the outcome measure for three consecutive sessions). Participant 11 also reached criterion for discontinuation for both treatments after only three sessions of the second treatment. Participant 9 was also a particularly strong responder to both treatments.

An examination of the strongest responders is more informative than one of the nonresponders. The three participants who responded most strongly to both treatments were motivated, reported being aware that their voices were not the same as before the stroke, and were judged to exhibit mild-to-moderate expressive aprosodia. Two of these three participants were also amateur musicians. Clinicians are probably not surprised by the possible prognostic significance of motivation, awareness, and relative mildness of symptoms. Nonetheless, confident and statistically based conclusions about response predictors will require treatment of a larger number of participants. In addition, this larger number of participants will require more sensitive measures of depression, insight, lesion volume and locus, and nature and severity of prosodic abnormality.

Also awaiting more data is a conclusion about which treatment has a larger effect. Both treatments showed treatment effects for most participants. However, the treating clinicians, all experienced SLPs, reported that the imitative treatment seemed easier for the participants to grasp quickly and often produced more immediate effects. On the other hand, they also reported that the cognitive-linguistic treatment appeared to carry over more readily to untreated utterances that involved the treated emotions. Regardless of these impressions, the data suggest that the first treatment was more likely to show a greater treatment effect than the second, regardless of which treatment was administered first. This was true for 8 of the 11 participants who received both treatments. However, in no case did a participant respond to the first treatment and not to the second.

The failure of the imitative treatment to generate larger treatment effects than the cognitive-linguistic treatment is somewhat surprising because the treatments differ in several ways that would seemingly favor the imitative approach. The first difference is the type of clinician cueing, which is primarily verbal in the imitative treat- ment and primarily written and pictured in the cognitivelinguistic treatment. As such, the imitative treatment more closely resembled the successful treatments reported by Anderson et al. [3] and Stringer [4]. A second difference is that participants had more opportunities for a verbal response in the imitative treatment. Heuristically, this greater opportunity for practice would seemingly favor the imitative treatment. A third difference is that the imitative treatment ended with a role-playing step that was not included in the cognitive-linguistic treatment. This more functional step would seem to be an additional strength. The most parsimonious explanations for the imitative treatment's failure to distance itself from the cognitive-linguistic treatment are the number and characteristics of the participants and the weaknesses of the ABAC design, issues to which we will return.

The lack of generalization to the randomly selected, untreated emotion (fear) was disappointing. This failure is not easily explained. Admittedly, fear was more challenging to judge but judgment reliability was acceptably high. Expressions of fear can vary by context, for example, when one is fearful and trying to be quiet compared with when one reacts to a surprising turn of events with a fearful utterance. However, this can be argued for the other emotions as well. Some evidence exists that positive emotions, such as happy, and negative emotions, such as anger, are served by different neural networks. However, the successfully treated emotions were both positive and negative. In our subsequent research, each participant's untreated emotion will be selected randomly. In addition, this failure of generalization will motivate continued improvement of the treatments.

Both treatments were theoretically motivated, and the a priori assumption was that the imitative approach would have the most robust effects. Aprosodia, especially in individuals who exhibit more expressive than receptive deficits, was hypothesized to implicate a higher order programming/planning deficit, and a more robust effect of the imitative treatment was predicted for these individuals. Participants were judged to have more severe expressive than receptive deficits if receptive and expressive performance differed by two scale points (normal, mild, moderate, severe). Four participants (participants 1, 5 , 6, and 14) met this criterion, but none responded more strongly to the imitative treatment.

A more anterior localization of lesions might more likely implicate programming impairment and a more robust response to the imitative treatment. This expectation 
also went unfulfilled. For example, participant 3 had a right medial frontal and supplementary motor area lesion, which might be expected to disrupt programming and planning, but had essentially equal responses to both treatments. Participant 11 had a more posterior lesion, which might reasonably be expected to influence an affect lexicon; however, his response to the imitative treatment was more substantial than to the cognitivelinguistic treatment. A larger number of participants with circumscribed lesions may eventually provide insight into the nature of the underlying pathophysiology in aprosodia.

An equally fruitful conceptualization for subsequent treatment studies of aprosodia is implicit and explicit memory. As Boyd and Winstein note [17], at least one form of implicit memory involves motor processes, while explicit memory involves facts and ideas. Rehabilitationists are increasingly concerned about the interaction of these memory systems in the reacquisition and maintenance of skills damaged by stroke. Depending on the kind and the timing of the information provided, explicit learning may influence motor performance [17]. The cognitive-linguistic treatment in the present study depended heavily on explicit memory because the treatment provided information about how emotional prosody was to be achieved. The imitative therapy depended more heavily on implicit memory because participants imitated the clinician's productions for much of the treatment. Some explicit knowledge emerged, of course, because knowledge of results was provided after every production. The potential influence of explicit and implicit memory systems on the acquisition and reacquisition of performance following stroke is an appealing guide for the next generation of aprosodia rehabilitation studies. Combining elements of the imitative and cognitivelinguistic treatments would potentially exploit the implicitexplicit interaction.

The design of subsequent studies also requires refinement. The ABAC design has weaknesses. Perhaps the major weakness is that treatment effects from the first treatment are likely to influence response to the second. In the original design, a 1-month nontreatment period occurred between the two treatments. This strategy, borrowed from the drug treatment literature, is problematic, especially for behavioral treatments. Performance had not returned to pretreatment levels prior to the second treatment. Indeed, for four of the six participants with 3-month follow-up data, performance had still not returned to pretreatment baseline. The best designs for behavioral treatments are withdrawal, multiple baseline, or randomized group designs. Our subsequent studies will use the latter.

\section{CONCLUSIONS}

Our results support the notion that expressive aprosodia following brain damage can be improved by treatment. However, much remains to be done. A treatment with even more robust treatment and generalization effects must be developed. Devising and testing such a treatment are our group's next research goals.

\section{ACKNOWLEDGMENTS}

This material was based on work supported in part by the Department of Veterans Affairs Office of Research and Development, Rehabilitation Research and Development Service, Brain Rehabilitation Research Center; and the National Institutes of Health, National Institute on Deafness and Other Communication Disorders, grant P50 DCO3888.

The authors have declared that no conflicting interests exist.

\section{REFERENCES}

1. Tucker DM, Watson RT, Heilman KM. Discrimination and evocation of affectively intoned speech in patients with right parietal disease. Neurology. 1977;27(10):947-50. [PMID: 561908]

2. Ross ED. The aprosodias. Functional-anatomic organization of the affective components of language in the right hemisphere. Arch Neurol. 1981;38(9):561-69. [PMID: 7271534]

3. Anderson JM, Beversdorf DQ, Heilman KM, Gonzalez Rothi LJ. Treatment of expressive aprosodia associated with right hemisphere injury. J Int Neuropsychol Soc. 1999;5:157.

4. Stringer AY. Treatment of motor aprosodia with pitch biofeedback and expression modeling. Brain Inj. 1996;10(8): 583-90. [PMID: 8836515]

5. Rosenbek JC, Crucian GP, Leon SA, Hieber B, Rodriguez AD, Holiway B, Ketterson TU, Ciampitti MZ, Heilman KM, Gonzalez Rothi LJ. Novel treatments for expressive aprosodia: a phase I investigation of cognitive linguistic 
JRRD, Volume 43, Number 3, 2006

and imitative interventions. J Int Neuropsychol Soc. 2004; 10(5):786-93. [PMID: 15327724]

6. Leon SA, Rosenbek JC, Crucian GP, Hieber B, Holiway B, Rodriguez AD, Ketterson TU, Ciampitti MZ, Freshwater S, Heilman KM, Gonzalez Rothi LJ. Active treatments for aprosodia secondary to right hemisphere stroke. J Rehabil Res Dev. 2005;42(1):93-101. [PMID: 15742253]

7. Van der Merwe A. A theoretical framework for the characterization of pathological speech sensorimotor control. In: McNeil MR, editor. Clinical management of sensorimotor speech disorders. New York (NY): Thieme Medical Publishers; 1997. p. 1-26.

8. Bowers D, Bauer RM, Heilman KM. The nonverbal affect lexicon: theoretical perspectives from neuropsychological studies of affect perception. Neuropsychology. 1993;7(4): 433-44.

9. Folstein MF, Folstein SE, McHugh PR. "Mini-mental state." A practical method for grading the cognitive state of patients for the clinician. J Psychiatr Res. 1975;12(3):189-98. [PMID: 1202204]

10. Lezak MD. Neuropsychological assessment. 3rd ed. New York (NY): Oxford University Press; 1995.

11. Benton AL, Hamsher K, Varney NR, Spreen O. Contributions to neuropsychological assessment. New York (NY): Oxford University Press; 1983.
12. Yesavage JA, Brink TL, Rose TL, Lum O, Huang V, Adey M, Leirer VO. Development and validation of a geriatric depression screening scale: a preliminary report. J Psychiatr Res. 1982-83;17(1):37-49. [PMID: 7183759]

13. Bowers D, Blonder L, Heilman KM. The Florida Affect Battery. Gainesville (FL): University of Florida Brain Institute; 1998.

14. Tryon WW. A simplified time-series analysis for evaluating treatment interventions. J Appl Behav Anal. 1982; 15(3):423-29. [PMID: 7142062]

15. Robey RR, Schultz MC, Crawford AB, Sinner CA. Singlesubject clinical-outcome research: designs, data, effect sizes, and analyses. Aphasiology. 1999;13(6):445-73.

16. Cohen J. Statistical power analysis for the behavioral sciences. 2nd ed. Hillsdale (NJ): Lawrence Erlbaum Associates; 1988.

17. Boyd LA, Winstein CJ. Implicit motor-sequence learning in humans following unilateral stroke: the impact of practice and explicit knowledge. Neurosci Lett. 2001;298(1): 65-69. [PMID: 11154837]

Submitted for publication January 28, 2005. Accepted in revised form June 24, 2005. 